\title{
Diagnose laboratorial das hepatopatias: debate acerca das fronteiras do processo de aprendizagem no ensino médico
}

\author{
Raniery Ávila de Oliveira \\ Mestrando em Ensino em Ciências da Saúde e do Meio Ambiente pelo Centro Universitário de Volta Redonda- \\ UNIFOA. Médico Gastroenterologista. Pós graduado em Hepatologia. \\ Sócio Titular da Sociedade Brasileira de Hepatologia e da Federação Brasileira de Gastroenterologia. \\ Preceptor do Décimo Primeiro Período do Curso de Medicina do UNIFOA. \\ $\checkmark$ rany-med@bol.com.br \\ Marcelo Paraiso Alves \\ Doutor em Educação pela Universidade Federal Fluminense- Niterói- RJ. Mestre em História pela USS. \\ Especialista em Educação Motora pelo UNIFOA. Licenciatura plena ampliada em Educação Física. \\ Integrante do grupo de pesquisa Cotidiano Escolar e Currículo. \\ Docente no Mestrado em Ciências da Saúde e do Meio Ambiente no Centro Universitário de Volta Redonda - \\ UNIFOA. \\ $\triangle$ marceloparaiso@outlook.com
}

\begin{abstract}
Resumo:
O presente ensaio objetiva discutir o processo de ensino e aprendizagem do diagnóstico laboratorial das doenças hepáticas e seu redimensionamento, tendo por paradigma o pensamento complexo. Nessa perspectiva, a revisão bibliográfica foi realizada nas principais bases de dados, com análise dos trabalhos, cuja temática relacionavase ao escopo do ensaio. O diagnóstico laboratorial em hepatologia é um processo fluído, que necessita ultrapassar os muros disciplinares impostos pela categorização das especialidades médicas. Outrossim, a complexidade inserida no ato de diagnosticar tem por base uma racionalidade que implica em prudência e atenção, e deve ser desvinculada da aparente relação dicotômica naturalizada pelo paradigma mecanicista. Repensar o modo como se ensina e como se aprende no processo de graduação em medicina é um aspecto prioritário, podendo impactar em mudanças de posturas necessárias à sociedade. Cabe frisar que tal proposição visa não apenas a assimilação do conteúdo, mas o desenvolvimento da competência médica para estabelecer interconexões com os demais campos do saber médico, bem como com os determinantes sociais, políticos, econômicos e culturais, atribuindo novos sentidos ao conhecimento. Assim, espera-se que esse debate acerca do diagnóstico hepático contribua com as discussões curriculares das escolas médicas, no intuito de promover a revisão de ementas, estratégias, metodologias e reflexões sobre o paradigma que subsidia os aspectos mencionados. Nessa lógica, a transversalidade pode estruturar mudanças no campo do conhecimento acerca do diagnóstico laboratorial das doenças hepáticas mais prevalentes, promovendo conhecimento mais pertinente, contextualizado, globalizado, complexo e capaz de refletir a pluralidade de saberes da sociedade contemporânea.
\end{abstract}

Palavras-chave: Educação médica, hepatologia, diagnóstico, currículo.

\section{Laboratory diagnosis of hepatopathies: debate about the borders of the learning process in medical education}

\begin{abstract}
:
This essay aims to discuss the teaching and learning process of the laboratory liver diseases diagnosis and their resizing, using complex thinking as a paradigm. In this perspective, the bibliographic review was carried out in the main databases, with analysis of the works, whose theme was related to the scope of the essay. Laboratory diagnosis in hepatology is a fluid process, which needs to overcome the disciplinary walls imposed by the categorization of medical specialties. Furthermore, the complexity inserted in the act of diagnosing is based on a rationality that implies prudence and attention, and must be disconnected from the apparent dichotomous
\end{abstract}


relationship naturalized by the mechanistic paradigm. Rethinking the way we teach and learn in the medical graduation process is a priority aspect, which can impact changes in attitudes necessary for society. It should be noted that this proposition aims not only to assimilate the content, but also to develop medical competence to establish interconnections with other fields of medical knowledge, as well as with social, political, economic and cultural determinants, giving new meanings to knowledge. Thus, it is expected that this debate about hepatic diagnosis contributes to the curricular discussions of medical schools, in order to promote the revision of menus, strategies, methodologies and reflections on the paradigm that supports the mentioned aspects. In this logic, transversality can structure changes in the field of knowledge about the laboratory diagnosis of the most prevalent liver diseases, promoting more pertinent, contextualized, globalized, complex knowledge and capable of reflecting the plurality of knowledge in contemporary society.

Keywords: Skills, physiotherapy, intensive care units, curriculum guidelines.

\section{Perfil de las habilidades del fisioterapeutas que actúan en unidades de terapia intensiva en el estado de Paraná}

\section{Resumen:}

El fisioterapeuta se incorporó a los equipos básicos de las unidades de cuidados intensivos (UCI) en 1998. Aunque las actividades de estos profesionales en estas unidades ya están reguladas y se ha demostrado su eficacia terapéutica, su identidad y reconocimiento parecen no estructurados hasta la fecha, lo que sugiere su construcción. en curso, a través de la identificación de atribuciones y competencias. El objetivo de este estudio es identificar el perfil de competencia de los fisioterapeutas que trabajan en UCI y relacionarlo con las competencias señaladas por las Pautas Curriculares Nacionales del curso de Fisioterapia. El estudio realizado es un tipo de encuesta, con un enfoque cuantitativo que utilizó un cuestionario estructurado, elaborado a partir del análisis del PCN del curso de fisioterapia, que fue enviado a 75 fisioterapeutas que trabajan en las UCI de 16 hospitales en Paraná, con un rendimiento del $66,6 \%$. Como resultado, apunta a un profesional que afirma tener habilidades esenciales, que actúa y reacciona con pertinencia frente a sus actividades con éxito profesional, de una manera valiente y ética, además de garantizar su autonomía y seguridad. Sin embargo, estos profesionales sugieren no adquirir estas cualidades en la educación básica, durante su graduado universitario. También indican que las habilidades señaladas por los PCN, a pesar de ser reales y vivas para ellos, pueden no ser consideradas en los cursos de pregrado, ya que no parecen ser obtenidas durante la graduación, sino después del comienzo de su actividad laboral. En conclusión, las respuestas de los fisioterapeutas muestran la necesidad del desarrollo continuo de habilidades, la importancia de la participación del fisioterapeuta en la UCI y la posibilidad de actuar con el apoyo de una formación profesional de excelencia.

Palabras clave: Competencias, fisioterapia, unidades de cuidados intensivos, pautas curriculares.

\section{INTRODUÇÃO}

O presente ensaio se delineia como um conhecimento de fronteira, porque é pensado no diálogo entre o campo do Ensino (Educação) e da Saúde, especificamente da Medicina. De outra maneira, ainda entendemos que as reflexões propostas nesse trabalho dialogam com outras epistemologias para além daquelas fundadas no paradigma científico-positivista e, portanto, na matriz anátomo-biológica, que desconsidera outras dimensões do ser humano: 
o estado emocional, a história de vida e familiar ou a situação social e econômica do paciente (NAKASU, 2013).

Considerando tais pressupostos, podemos afirmar que o presente estudo está a caminho do Sul, pois entende que a dicotomia Norte-Sul, expressa pelas linhas abissais (capitalismo, colonialismo e patriarcado), legitima uma forma de racionalidade que cria e sustenta desigualdades sociais no sistema mundial. Assim, vamos entender fronteira como uma metáfora, uma figura que, se por um lado pode legitimar a racionalidade dominante (Paradigma Moderno), por outro, reconhece a existência de subjetividades, de sociabilidades, culturas, epistemologias outras, e, por que não dizer, alternativas que permitem a construção de outros paradigmas fundados nos princípios da pluralidade, solidariedade e da participação coletiva (SANTOS, 2018).

Portanto, estamos a afirmar que a formação médica vem sendo rediscutida com vistas a adotar um currículo adequado à realidade dos sistemas de saúde, uma vez que podemos encontrar pelo menos três momentos em sua história em que o Estado visa a garantir o acesso à saúde: início do século XX, viabilizado por campanhas contra epidemias em regiões de interesse comercial. A esse respeito, entendemos que tal paradigma foi responsável pela instauração do olhar reducionista de educação, possibilitando a ampliação de escolas de medicina que condicionavam o(a) estudantes a conceber, exclusivamente, sua intervenção clínica via perspectiva anatomobiológica de seus pacientes" (MEIRELES, 2019). No segundo momento, o Estado, via pecúlio obrigatório, procurou garantir o acesso aos trabalhadores até meados da década de 1980, quando entrou em colapso. E, por fim, pós-Constituição de 1988, quando a saúde passou a ser concebida como um direito (MORETTI-PIRES; BUENO, 2009).

Diante do exposto, cabe refletir: A transversalidade como um paradigma que procura romper com o reducionismo cartesiano poderia contribuir com o processo de ensinoaprendizagem no diagnóstico laboratorial das doenças hepáticas?

Sabemos do desafio atual pela busca de alternativas que possam contribuir para a formação do profissional necessário à sociedade contemporânea. Acerca disso, destacam-se as perspectivas sociais dos papéis de professor e estudante, que vêm se remodelando ao longo das gerações, com o surgimento de novas metodologias e abordagens pedagógicas distintas, determinando novas formas de ensinar e aprender (D'COSTA; SWARNADAS, 2016). 
Ainda com referência ao processo de ensinar e aprender, a transdisciplinaridade pode ser considerada como uma abordagem pedagógica científica, que visa à unidade do conhecimento, procurando estimular uma nova compreensão da realidade, articulando os elementos que perpassam entre, além e através das disciplinas (MORIN, 2002).

Assim, no presente ensaio, objetivamos discutir o processo de ensino e aprendizagem das doenças hepáticas e seu redimensionamento, via pensamento complexo. Tal ideia considera a possibilidade de potencializar o ato de ensinar e aprender viabilizado pelo diagnóstico laboratorial. O que buscamos debater emerge da perspectiva que concebe a hepatologia como um espaço complexo que necessita ultrapassar os muros disciplinares, uma vez que essa área transcende o limite imposto pela categorização das especialidades médicas. Pensar nessa direção nos remete a conceber o fígado como um laboratório químico, e sede de todo metabolismo intermediário, pois a sua função enreda-se a vários efeitos, tanto como causa, quanto consequência. Portanto, diagnóstico laboratorial das hepatopatias é uma entidade fluída e complexa, que não pode se isolar de todo o contexto clínico do qual faz parte. Por isso a importância da analogia adotada no presente artigo entre o diagnóstico laboratorial das doenças hepáticas e o paradigma complexo.

\section{A EDUCAÇÃo MÉdICA E A PERSPECTIVA DE MUDANÇA PARADIGMÁTICA}

É impossível discutir a educação médica sem referir Flexner e seu relatório. As forças e ideias sobre a educação médica existentes naquele momento histórico nos EUA e na Alemanha foram habilmente utilizadas por ele, tornando-o "líder autoproclamado do movimento de reforma do ensino médico, que mais tarde o tornaria publicamente reconhecido" (KEMP; EDLER, 2004).

A avaliação proposta por Flexner foi o principal, e praticamente único, instrumento para acreditação das escolas médicas nos Estados Unidos e Canadá, com implicações diretas em todo o mundo ocidental durante a primeira metade do século XX (HIATT, 1999).

O trabalho de Flexner (PAGLIOSA; DA ROS, 2008) permitiu reorganizar e regulamentar o funcionamento das escolas médicas. Mas, ao focar toda a sua atenção no aspecto biologicista 
e mecanicista, desconsiderou outros fatores que afetam profundamente os impactos da educação médica na prática profissional e na organização dos serviços de saúde.

Esta linha de pensamento (BARROS, 2014) nos chama a atenção para o fato de que o Relatório Flexner, apesar de promover significativo processo de transformações associadas ao processo de saúde-doença-cuidado, apenas deixa transparecer a necessidade de privilegiar o aspecto social na formação e trabalho médico.

Face ao exposto, torna-se fundamental a ruptura com o paradigma positivista que concebe o corpo sob a ótica mecanicista, uma vez que concebia o mundo e o homem como um aparato anátomo-biológico. $\mathrm{O}$ paradigma cartesiano rejeita qualquer forma de saber subjetivo, pois considera apenas o caráter cientificista, palpável e quantificável, do mesmo que o pensamento integralizador nega a dicotomização ao se deparar com os problemas de saúde, onde o profissional se responsabiliza por uma parcela do indivíduo (SANTOS; RIBEIRO; ANJOS; FARIAS; LUCENA, 2015).

Nessa perspectiva, remetemos a ideia de que, durante a graduação médica, novas formas de ensinar e aprender necessitam ser repensadas, inclusive, considerando o escopo do presente ensaio. Para tanto, é fundamental um trabalho de descentramento: "Estabelecer uma relação entre saberes contentando-se em apreender um mesmo objeto de diversos pontos de vista, para fazer com que o aluno dê conta concretamente da quantidade de olhares possíveis que se pode dirigir a um objeto, fazendo com que as diferentes ciências que apreendem esse objeto, possam juntar-se ou desconjuntar-se" (LERBERT, 2002), o que obriga a mergulhar naquilo que foi adquirido num quadro mais vasto que conduza a certa relativização (MORIN, 2002).

\section{PENSAMENTO COMPLEXO E GRADUAÇÃO MÉDICA: CONTRIBUIÇÕES NA DIAGNOSE LABORATORIAL EM HEPATOLOGIA}

Ao refletir sobre a práxis na Educação Médica, especificamente enredado ao processo de ensino e aprendizagem no décimo primeiro módulo do curso de Graduação em Medicina (Internato de Saúde Coletiva), identificamos que a ótica cartesiana influencia significativamente o modo como os graduandos de Medicina concebem a construção do 
conhecimento. Tal percepção emerge dos questionamentos dos estudantes ao se depararem com a necessidade de interpretar os dados dos exames laboratoriais, conforme podemos verificar: Para que serve o que estou estudando? Como ligar esse conteúdo à minha prática, visto que farei cirurgia e não seguirei área clínica?

Diante de tais interrogações, fica explícita a ausência de interconexões entre os conhecimentos adquiridos em outros módulos e que, nesse momento de sua formação, seriam fundamentais para acessar e interpretar os dados dos exames laboratoriais das doenças hepáticas.

Todavia, se desejarmos que os graduandos utilizem os conhecimentos científicos adquiridos, torna-se necessário compreender o paradigma científico que está na base de seu processo de formação e que, de certo modo, ampara à sua maneira de pensar, sentir e agir no mundo. Isso nos remete à necessidade de promover outras experiências educativas capazes de provocar outras racionalidades, proporcionando novos significados durante o processo de ensino e aprendizagem do diagnóstico laboratorial das doenças hepáticas. Portanto, se por um lado o estudante é incitado, pelo docente, a empilhar os conhecimentos, seguindo a lógica da educação bancária (FREIRE, 2013), por outro lado, esses estudantes consideram essa perspectiva suficiente, permitindo-lhes progredir, tornando-os precisos nos seus respectivos campos de atuação.

Diante deste dilema existe uma eufemização da ciência. Com muita frequência vai-se de um problema a um resultado por um caminho bem construído que ninguém questiona. Sendo isso vivenciado, ao se ensinar somente de forma expositiva, durante o curso médico, os métodos de diagnóstico laboratorial das doenças hepáticas mais prevalentes.

Outro ponto de destaque é quanto à maneira de ensinar e aprender. Na hepatologia há uma diversidade de conteúdo a ser apreendido pelo médico em formação generalista. 0 sistema pedagógico baseado no consumo dos saberes não permite que o conhecimento seja suficientemente interiorizado, dificultando ao estudante ter uma capacidade de descentramento.

Diante do exposto, salientamos a necessidade de se proclamar, na graduação médica, outro paradigma que permita transformar o processo de ensino e aprendizagem das doenças 
hepáticas, e seus respectivos diagnósticos laboratoriais, como parte de uma produção de conhecimento, e não somente no consumo quantitativo do saber.

o que estamos a defender é a instauração do pensamento complexo, e consequentemente da ótica transdisciplinar, como possível paradigma capaz de promover a ampliação da percepção do estudante diante do conhecimento apreendido, por ser necessário não apenas assimilar o conteúdo, mas ser capaz de estabelecer interconexões com os demais campos do saber médico e os determinantes sociais, políticos, econômicos, culturais, de raça, contribuindo na atribuição de novos sentidos ao conhecimento.

Para Estrada (2009), a questão paradigmática ultrapassa os pressupostos epistemológicos e metodológicos, visto que está enredado ao modo como estabelece a leitura da realidade (gnoseologia) e natureza dessa mesma realidade (ontologia).

Nessa linha de pensamento, Estrada (2009) afirma que, ao fundar as reflexões a partir das "noções de pluralidade e complexidade dos sistemas físicos, biológicos e antropossociológicos, cuja compreensão requer outro paradigma - o da complexidade", torna-se fundamental operar com uma racionalidade aberta fundada sob um viés que se caracteriza por ser "evolutiva, residual, complexa e dialógica" (ESTRADA, 2009).

Entender esse processo sob a ótica do pensamento complexo pode, neste momento, auxiliar de forma crítica o redimensionamento do ensinar e aprender diagnóstico laboratorial das doenças hepáticas.

No ato complexo de ensinar e aprender diagnóstico laboratorial há a insurgência do Princípio Dialógico. Há um jogo entre a ordem, a desordem e a organização. Assim, para que se efetue diagnóstico laboratorial de uma doença hepática é necessário que além de conhecimentos de anatomia, fisiologia, bioquímica, histologia, sejam considerados os contextos clínicos, epidemiológicos e sociais, bem como as relações com os demais órgãos e sistemas, por vezes categorizados em outras especialidades: "Ele une dois princípios ou noções que devem excluir-se um ao outro, mas não são indissociáveis numa mesma realidade" (MORIN, 2000).

Nessa perspectiva, o poder da nossa lógica não é absoluto, para pensar é preciso ser capaz de transgredi-la e depois voltar a ela, fazendo verificações segmentárias, pois o Princípio Hologramático coloca em evidência o paradoxo "em que não somente a parte está 
no todo, mas em que o todo está inscrito na parte" (MORIN, 2000), o que nos remete a outro princípio - do Círculo Retroativo - que, ao romper com a linearidade cartesiana, opera com uma ótica que concebe causa e efeito como sistema de retroalimentação: "as retroações são legiões de fenômenos econômicos, sociais, políticos e psicológicos" (MORIN, 2000).

Desse modo, para que um conteúdo seja considerado pertinente, será preciso reunir, contextualizar, globalizar, interconectar nossas informações e nossos saberes, buscar, portanto, um conhecimento complexo. Em outro sentido, a consciência da complexidade nos faz compreender que jamais poderemos escapar da incerteza e que jamais poderemos ter um saber total.

Ao inferirmos ao raciocínio clínico o pensamento complexo, não estamos recusando a clareza, o determinismo e a ordem. Apenas os considerando insuficientes para programar o diagnóstico, o conhecimento, e, consequentemente, as ações.

Em contrapartida, a concepção mecanicista, ainda presente, permanece exercendo uma influência na graduação médica e isso precisa ser revisto. A fragmentação das disciplinas acadêmicas que impedem estabelecer o vínculo entre as partes e o todo, aparenta uma relação contraditória e incoerente com a aprendizagem do diagnóstico laboratorial em hepatologia. Em relação a isto, Morin (2002) alerta-nos dizendo que, de fato, a especialização (disciplinas) impede tanto a percepção do global que ela fragmenta em parcelas, quanto do essencial que ela dissolve. Impedindo até mesmo de tratar corretamente as patologias, que só podem ser compostas e pensadas em seu amplo contexto. Os problemas essenciais não são fragmentáveis, e os problemas globais são cada vez mais essenciais, por isso, faz-se necessário o exercício do conhecimento pertinente no próprio seio das escolas médicas.

Nesse sentido, o compromisso é estimular o discente a ressignificar o conteúdo. Ensinar e aprender na perspectiva da complexidade é considerar a indissociabilidade do conhecimento, favorecendo então a formação do médico necessário para sociedade moderna. 


\section{O DIAGNÓSTICO LABORATORIAL DAS HEPATOPATIAS E SUA RELEVÂNCIA}

Os sintomas e sinais clínicos das doenças hepáticas aparecem, em geral, tardiamente na história natural desses processos patológicos. Felizmente, existe um conjunto de testes diagnósticos que é capaz de detectar de forma precoce sua instalação. Além de permitir o diagnóstico, os testes hepáticos podem fornecer informações a respeito da gravidade, da lesão, do prognóstico e da terapia (MATTOS; DANTAS-CORRÊA, 2010).

É notório o excesso de solicitações de exames (ROCHA; LIMA; ALMEIDA; LOPES, 2018). E isso pode ter motivação em questões que envolvem desde a demanda do paciente atendido até questões, possivelmente, relacionadas ao deficit no processo de ensino-aprendizagem durante a graduação médica.

A solicitação injustificada de exames laboratoriais pode ter efeitos negativos tanto para o cuidado com o paciente, quanto para o sistema de saúde, o que corrobora para diagnósticos equivocados, para gastos de recursos desnecessários, para atraso em diagnósticos possíveis apenas com evidências clínicas (ROCHA; LIMA; ALMEIDA; LOPES, 2018).

Considerando o elevado custo com atenção à saúde, há uma redução nos atendimentos de parte da população, já que os recursos disponíveis são usados de maneira inadequada. A solicitação de exames de forma compulsiva, ou mesmo dispensável, também gera problemas pela necessidade de acompanhamento de um resultado alterado, sem relevância clínica. Outro dado de importância, enquanto verbas e tempo são mal gerenciados, a morbidade e letalidade de determinadas patologias crescem de forma exponencial ((ROCHA; LIMA; ALMEIDA; LOPES, 2018).

A complexidade inserida no ato de diagnosticar não é uma receita ou modelo para conhecer o inesperado, mas uma racionalidade que implica em prudência e atenção, que deve ser desvinculado dos determinismos e da aparente relação dicotômica imposta pelo paradigma mecanicista: "O conceito de ordem extrapola as ideias de estabilidade, rigidez, repetição e regularidade, unindo-se à ideia de interação, e imprescinde, recursivamente, da desordem, que comporta dois pólos: um objetivo e outro subjetivo" (ESTRADA, 2009). Para o autor (ESTRADA, 2009), o caráter objetivo emerge dos desvios que perturbam e geram transformações, podendo emergir dos acidentes, dos erros e das instabilidades. Já o polo subjetivo é marcado pela indeterminabilidade e traduz-se pelo princípio da incerteza 
(MORIN, 2003): “Acrescento que a auto-organização, característica dos fenômenos vivos, comporta permanente processo de desorganização transformado em processo permanente de reorganização, até a morte, evidentemente" (MORIN, 2003).

Nesse contexto, o diagnóstico em hepatologia é dotado de desafios. A dificuldade dos discentes em solicitar e interpretar exames laboratoriais para diagnóstico das doenças hepáticas é uma constatação. Sobretudo, a capacidade de diagnosticar não deve ser reduzida a eventos empíricos ou ilustrações pontuais, mas deve exigir uma intencional e contínua postura problematizadora da história de vida, da experiência, da observação e da discussão. Nessa lógica, o pensamento complexo auxiliará que "outros" conhecimentos adquiram novos sentidos e significados, potencializando o ensino-aprendizagem e a práxis do futuro profissional.

\section{CONSIDERAÇÕES FINAIS}

Repensar como se ensina e como se aprende durante a graduação médica é um meio necessário que pode impactar em mudanças de posturas necessárias à sociedade. 0 contexto da vida atual é dinâmico, reflexo de uma série de mudanças nos paradigmas pessoais, socioculturais e econômicos. Não considerar esse cenário coloca em risco as estratégias de promoção, prevenção e intervenção adotadas na perspectiva de saúde e doença.

Assim, as escolas médicas necessitam rever suas ementas, repensar suas estratégias. Nessa lógica, a transversalidade pode estruturar mudanças no campo do conhecimento acerca do diagnóstico laboratorial das doenças hepáticas mais prevalentes, promovendo conhecimento mais pertinente, contextualizado, globalizado, complexo e capaz de refletir acerca da pluralidade de saberes da sociedade contemporânea. 


\section{REFERÊNCIAS BIBLIOGRÁFICAS}

BARROS, N.F. O ensino das ciências sociais em saúde: entre o aplicado e o teórico. Ciência \& Saúde Coletiva, n. 19, v. 4, p. 1.053-1.063, 2014.

D' COSTA, M.P.; SWARNADAS, G.S. Students' perceptions of effective clinical teaching and teacher behaviours on learning. Manipal Journal of Nursing and Health Sciences, v. 2, p. 1-8, 2016.

ESTRADA, A.A. Os fundamentos da teoria da complexidade em Edgar Morin. Akrópolis-Revista de Ciências Humanas da UNIPAR, n. 17, v. 2, p. 85-90, 2009.

FREIRE, P. Pedagogia do Oprimido. Rio de Janeiro: Paz \& Terra, 2013.

HIATT, M.D. Around the continent in 180 days: The controversial journey of Abraham Flexner. The Pharos Alpha Omega Alpha Honor Med. Soc., n. 62, v. 1, p. 18-24, 1999.

KEMP, A.; EDLER, F.C. A reforma médica no Brasil e nos Estados Unidos: uma comparação entre duas retóricas. Hist. cienc. saúde-Manguinhos, n. 11, v. 3, p. 569-58, 2004.

LERBERT, G. Transversalidade e Educação. In: MORIN, E. A Religação dos Saberes. O desafio do século XXI. Rio de Janeiro: Bertrand Brasil, 2002.

MATTOS, A.A.; DANTAS-CORRÊA, E.B. Tratado de Hepatologia. Rio de Janeiro: Rubio, 2010.

MEIRELES, M.A.C.; FERNANDES, P.C.C.; SILVA, L.S. Diretrizes Curriculares Nacionais e a Formação Médica: Expectativas dos Discentes do Primeiro Ano do Curso de Medicina de uma Instituição de Ensino Superior. Rev. bras. educ. med., n. 43 , v. 2, p. 67-78, 2019.

MORETTI-PIRES, R.O.; BUENO, S.M.V. Freire e formação para o Sistema Único de Saúde: o enfermeiro, o médico e o odontólogo. Acta paul. enferm., n. 22, v. 4, p. 439-444, 2009.

MORIN, E. A Religação dos Saberes: O desafio do século XXI. 3. ed. Rio de Janeiro: Bertrand Brasil, 2002.

MORIN, E. Ciência com consciência. 7. ed. Rio de Janeiro: Bertrand Brasil, 2003.

MORIN, E. O método 2: a vida da vida. Porto Alegre: Sulina, 2001.

MORIN, E.; LE MOIGNE, J.L. A inteligência da complexidade. São Paulo: Petrópolis, 2000.

NAKASU, M.V.P. Em defesa de uma certa enfermidade: cuidados paliativos em debate. Revista Ciências em Saúde, $n$. 3, v. 4, p. 2-8, 2013.

PAGLIOSA, F.L.; DA ROS, M.A. O Relatório Flexner: Para o Bem e Para o Mal. Rev.bras. educ. méd., n. 32, v. 4, p. 492499, 2008.

ROCHA, E.C.B.; LIMA, L.J.L.; ALMEIDA, M.V.G.; LOPES, M.R. Necessidade de gerenciamento dos gastos com exames laboratoriais no Brasil. Revista de Educação da Universidade Federal do Vale do São Francisco, n. 8, v. 15, p. 112128, 2018.

SANTOS, B.S. Construindo as Epistemologias do Sul: Antologia Esencial. Volume I: Para um pensamento alternativo de alternativas. Ciudad Autónoma de Buenos Aires: CLACSO, 2018.

SANTOS, R.N.L.C.; RIBEIRO, K.S.Q.S.; ANJOS, U.U.; FARIAS, D.N.; LUCENA, E.M.F. Integralidade e Interdisciplinaridade na formação de estudantes de medicina. Rev. bras. educ. méd., n. 39, v. 3, p. 378-387, 2015.

\section{$(\mathrm{cc}) \mathrm{Br}$}

Este trabalho está licenciado com uma Licença Creative Commons - Atribuição 4.0 Internacional. 